01

\title{
Влияние асимметричного переноса зарядана ИК спектры возбужденных квадрупольных молекул
}

\author{
(C) В.Г. Ткачёв, А.И. Иванов \\ Волгоградский государственный университет, \\ 400062 Волгоград, Россия \\ ฯ e-mail: Anatoly.Ivanov@volsu.ru \\ Поступила в редакцию 07.05.2020 г. \\ В окончательной редакции 01.07.2020 г. \\ Принята к публикации 07.07.2020 г.
}

Исследовано влияние нарушения симметрии переносом заряда в возбужденных квадрупольных молекулах вида D- $\pi-\mathrm{A}-\pi$-D или A- $\pi$-D- $\pi$-A, где A и D - электронно-акцепторная и электронно-донорная группы, на нестационарные спектры характеристических колебаний. В рамках разработанной ранее теории нарушения симметрии и его проявления в нестационарных ИК спектрах дана классификация типов возможных изменений ИК спектров с ростом степени асимметрии молекул. Представлена физическая интерпретация выявленных закономерностей и проведено их сравнение с имеющимися в настоящее время экспериментальными данными.

Ключевые слова: времяразрешенные ИК спектры, электронно-колебательное взаимодействие, нарушение симметрии переносом электрона, взаимодействие колебательных мод.

DOI: $10.21883 /$ OS.2020.11.50159.149-20

\section{Введение}

Молекулы вида D- $\pi$-A- $\pi$-D (A- $\pi$-D- $\pi$-A), где А и D электронно-акцепторная и электронно-донорная группы, обладающие центром инверсии, в силу своей симметрии имеют нулевой дипольный и большой квадрупольный моменты. Такие квадрупольные молекулы первоначально привлекли внимание исследователей большим сечением двухфотонного поглощения, что важно для ряда перспективных приложений [1-3]. Позже было обнаружено, что спектры поглощения этих молекул, как и ожидалось, слабозависят от полярности растворителя, тогда как спектры флуоресценции демонстрируют сильный сольватохромный сдвиг [4-7]. Такое поведение было интерпретировано как нарушение симметрии в возбужденном состоянии асимметричным переносом заряда с центральной группы на периферийные, приводящим к формированию значительного дипольного момента $[8,9]$. Применение методов инфракрасной спектроскопии с фемтосекундным временным разрешением позволило получить не только веские свидетельства нарушения симметрии, но и зарегистрировать динамику нарушения симметрии [10-13]. Для данного класса молекул ИК спектроскопия характеристических локализованных колебаний обладает внутримолекулярным пространственным разрешением в отличие от оптической. В экспериментах исследовались молекулы, включающие $-\mathrm{C} \equiv \mathrm{N}$ или $-\mathrm{C} \equiv \mathrm{C}$-группы в обеих ветвях. Валентные колебания этих групп чувствительны к распределению электронной плотности в молекуле, поэтому частоты валентных колебаний этих групп в разных ветвях молекулы должны совпадать в симметричном состоянии и различаться в асимметричном состоянии, что и наблюдалось в экспериментах [10-13].

Электронные состояния квадрупольных молекул в силу их инверсионной симметрии имеют определенную четность. Поскольку основное состояние является четным, то франк-кондоновское состояние, формируемое оптическим коротким импульсом накачки, является нечетным. Выше этого нечетного состояние расположено четное, которое проявляется в двухфотонном поглощении. Непосредственно после формирования нечетного состояния ИК спектр содержит одну полосу, соответствующую антисимметричным валентным колебаниям

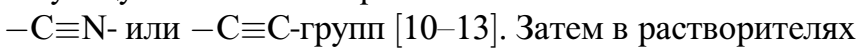
может появляться вторая полоса. Время формирования квазистационарного ИК спектра лежит в пикосекундной области и коррелирует с временем релаксации растворителя.

Значение расщепления ИК полос в асимметричном состоянии очень чувствительно к взаимодействию молекулы с растворителем $[11,13]$. Например, в молекуле, состоящей из ядра пирролопиррола D и двух акцепторов цианофенила (в дальнейшем в ссылках на эту молекулу используется обозначение A- $\pi$-D- $\pi$-A), расщепление монотонно возрастает с ростом полярности растворителя и увеличивается далее при образовании водородных и галогеновых связей [13]. Учитывая высокую точность измерения этого расщепления, открывается возможность создания перспективной шкалы энергии межмолекулярных взаимодействий.

Оптические спектры квадрупольных молекул были интерпретированы в рамках „модели существенных состояний“, включающей основное состояние и два ниж- 
них возбужденных вырожденных состояния с локализацией заряда на разных плечах молекулы [14]. В рамках этой модели было описано нарушение симметрии асимметричным переносом электрона с центра на ветви и воспроизведены особенности одно- и двухфотонных электронных спектров [14]. Недавно для описания нарушения симметрии в квадрупольных молекулах была предложена двухуровневая модель, включающая только два возбужденных вырожденных цвиттер-ионных состояния [15]. В отличие от „модели существенных состояний эта модель имеет аналитическое решение, устанавливающее связь степени нарушения симметрии с несколькими параметрами, имеющими ясный физический смысл. Область применимости модели ограничена условием: энергетическая щель между первым возбужденным и основным состояниями должна быть много больше энергетической щели между двумя нижними возбужденными состояниями [16]. Далее была разработана теория, связывающая форму ИК спектров с параметром асимметрии [17]. Эта теория хорошо описывает изменение спектров $-\mathrm{C} \equiv \mathrm{N}$-колебаний в растворителях различной полярности [18] и эволюцию спектров поглощения $-\mathrm{C} \equiv \mathrm{C}$-групп с ростом степени асимметрии [19].

Целями настоящей работы являются: (а) выявление типов возможных изменений нестационарных ИК спектров с ростом степени асимметрии, (б) физическая интерпретация выявленных закономерностей, (в) сопоставление полученных закономерностей с имеющимися в настоящее время экспериментальными данными.

\section{Нарушение симметрии переносом электрона в возбужденных квадрупольных молекулах и его влияние на колебательный спектр}

Простейшая модель нарушения симметрии в квадрупольных молекулах вида $\mathrm{A}_{\mathrm{L}} \mathrm{DA}_{\mathrm{R}} \mathrm{c}$ одинаковыми электронно-акцепторными группами $\mathrm{A}_{\mathrm{L}}$ и $\mathrm{A}_{\mathrm{R}}$ описана в [15], влияние нарушения симметрии на колебательный спектр молекулы представлено в [16,17]. В этом разделе мы дадим краткий обзор модели и определим физические величины, используемые в модели. Два базисных цвиттер-ионных состояния $\mathrm{A}_{\mathrm{L}}^{-} \mathrm{D}^{+} \mathrm{A}_{\mathrm{R}}$ и $\mathrm{A}_{\mathrm{L}} \mathrm{D}^{+} \mathrm{A}_{\mathrm{R}}^{-}$описываются волновыми функциями $\varphi_{\mathrm{L}}$ и $\varphi_{\mathrm{R}}$. В произвольном состоянии $\Psi=a_{\mathrm{L}} \varphi_{\mathrm{L}}+a_{\mathrm{R}} \varphi_{\mathrm{R}}$ заряды на акцепторах определяются выражениями $\delta_{\mathrm{L}}=e\left|a_{\mathrm{L}}\right|^{2}$ и $\delta_{\mathrm{R}}=e\left|a_{\mathrm{R}}\right|^{2}$. Амплитуды $a_{\mathrm{L}}$ и $a_{\mathrm{R}}$ предполагаются вещественными. Они определяют параметр асимметрии $D=a_{\mathrm{L}}^{2}-a_{\mathrm{R}}^{2}$. Отметим, что преобразование инверсии меняет знак $D$. Очевидно, что дипольный момент молекулы в состоянии с нарушенной симметрией равен $\mu_{0} D$, где $\mu_{0}$ - дипольный момент молекулы в цвиттер-ионных состояниях $\varphi_{\mathrm{L}}$ и $\varphi_{\mathrm{R}}$.

Дипольный момент молекулы в полярной среде определяется поляризацией среды и флуктуирует вместе со средой [20]. Характерные времена термических флуктуаций поляризации много больше периода внутримолекулярных высокочастотных колебаний, поэтому в адиабатическом приближении мы можем записать гамильтониан колебательной подсистемы молекулы при фиксированном значении $D$. Гамильтониан внутримолекулярных колебаний, включающий электронно-колебательное взаимодействие, имеет вид $[16,17]$

$$
H=H_{V 0}+U_{\text {int }},
$$

В гармоническом приближении гамильтониан колебательной подсистемы квадрупольной молекулы с ненарушенной симметрией записывается как

$$
H_{V 0}=\frac{1}{2} \sum_{i}\left[p_{s i}^{2}+\omega_{s i}^{2} x_{s i}^{2}\right]+\frac{1}{2} \sum_{j}\left[p_{a j}^{2}+\omega_{a j}^{2} x_{a j}^{2}\right],
$$

где $p_{s i}, p_{a j}, x_{s i}, x_{a j}, \omega_{s i}, \omega_{a j}-$ импульсы, координаты и частоты нормальных симметричных и антисимметричных колебаний соответственно. Электронноколебательное взаимодействие $U_{\text {int }}$ определяется через его разложение по степеням $D$ и координатам осцилляторов. Учитывая малость изменений спектра колебаний при нарушении симметрии, можно ограничиться вторым порядком. В результате получим [17]

$$
\begin{gathered}
U_{\mathrm{int}}=U_{\mathrm{int}}^{(1)}+U_{\mathrm{int}}^{(2)} \\
U_{\mathrm{int}}^{(1)}=D \sum_{j} \xi_{j} x_{a j}, \\
U_{\mathrm{int}}^{(2)}=D \sum_{i j} \delta_{i j} x_{a j} x_{s j}+\frac{D^{2}}{2} \sum_{i k}\left[\alpha_{i k} x_{a i} x_{a k}+\beta_{i k} x_{s i} x_{s k}\right] .
\end{gathered}
$$

В этом разложении учтено, что гамильтониан должен быть инвариантен относительно преобразования инверсии. Здесь $\xi_{j}$ - параметры линейного электронноколебательного взаимодействия. Параметры $\alpha_{i k}, \beta_{i k}$ и $\delta_{i j}$ описывают изменения колебательных частот и эффект Душинского.

Линейное электронно-колебательное взаимодействие при фиксированном $D$ не влияет на колебательный спектр. Тем не менее, оно имеет два важных проявления. Во-первых, оно наряду с взаимодействием дипольного момента с поляризацией среды и кулоновского взаимодействия зарядов $\delta_{\mathrm{L}}$ и $\delta_{\mathrm{R}}$ индуцирует нарушение симметрии $[14,15,17]$. Во-вторых, оно понижает частоты антисимметричных колебаний $\omega_{a j}^{2} \rightarrow \omega_{a j}^{2}-\zeta_{j}^{2} / V$ в состоянии без нарушенной симметрии [17], где $V-$ недиагональный матричный элемент полного гамильтониана системы, построенный на базисных функциях $\varphi_{\mathrm{L}}$ и $\varphi_{\mathrm{R}}$ и равный половине расщепления полос одно- и двухфотонного поглощения (рис. 1).

Квадратичное электронно-колебательное взаимодействие, будучи слабым, может смешивать только колебательные моды с близкими частотами. Это означает, что для описания спектра характеристических колебаний, 


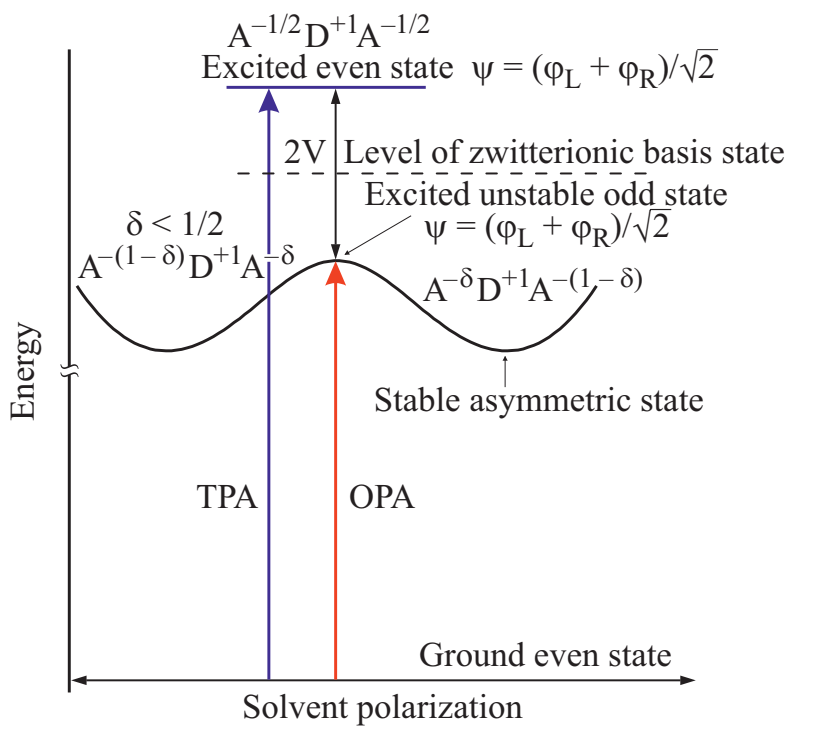

Рис. 1. Схема энергетических уровней квадрупольной молекулы A- $\pi$-D- $\pi$-A. Использованы сокращения ОРА и TPA для одно- и двухфотонного поглощения. В четном и нечетном состояниях дипольный момент молекулы равен нулю и ее энергия не зависит от поляризации среды. При нарушении симметрии возникает дипольный момент молекулы и поляризация среды приводит к понижению энергии системы. Штриховая горизонтальная линия соответствует уровню энергии цвиттерионного базисного состояния без взаимодействия молекулы со средой.

частоты которых отделены от всех остальных значительным интервалом, можно ограничиться приближением, в котором учитывается только взаимодействие симметричных и антисимметричных характеристических колебаний с близкими частотами. В результате в выражении для энергии электронно-колебательного взаимодействия (5) останется под знаками сумм по одному слагаемому, и в дальнейшем индексы $i, j, k$ опускаем.

Валентные колебания невзаимодействующих $-\mathrm{C} \equiv \mathrm{N}$ или $-\mathrm{C} \equiv \mathrm{C}$-групп, симметрично расположенных в левой и правой ветвях молекулы, описываются локальными координатами $x_{\mathrm{L}}$ и $x_{\mathrm{R}}$. Следует отметить, что здесь имеются в виду нормальные колебания, в которые основной вклад дают $-\mathrm{C} \equiv \mathrm{N}$ - или $-\mathrm{C} \equiv \mathrm{C}$-группы, но также вносят свой вклад и другие атомы молекулы. Истинными нормальными координатами квадрупольных молекул являются антисимметричная и симметричная комбинация локальных координат $x_{s / a}=\frac{1}{2}\left(x_{\mathrm{L}} \pm x_{\mathrm{R}}\right)$ колебаний с совпадающими частотами. Линейное электронноколебательное взаимодействие, как и ангармоническое взаимодействие мод, приводит к расщеплению частот антисимметричных и симметричных колебаний $\omega_{a}$ и $\omega_{s}[17]$.

Инфракрасный спектр возбужденной квадрупольной молекулы в состоянии с нарушенной симметрией рассчитывается в рамках классической теории поглощения электромагнитных волн осцилляторами. Исходными яв- ляются уравнения движения для колебательных мод под действием электромагнитной волны с частотой $\omega$ :

$$
\begin{gathered}
\ddot{x}_{a}+\eta_{a} \dot{x}_{a}+\frac{\partial H}{\partial x_{a}}=F \cos (\omega t), \\
\ddot{x}_{s}+\eta_{s} \dot{x}_{s}+\frac{\partial H}{\partial x_{s}}=0,
\end{gathered}
$$

где $\eta_{a}$ и $\eta_{s}-$ коэффициенты трения, определяющие однородную ширину полос поглощения, отражают конечное время жизни возбужденных состояний рассматриваемых колебательных мод, $F-$ амплитуда силы взаимодействия антисимметричного колебания с электромагнитной волной. Здесь учтено, что с электромагнитной волной взаимодействует только антисимметричное колебание.

Решение системы уравнений (6), (7) приводит к следующему выражению для спектра поглощения [17]:

$$
I(\omega)=-2 \omega F \operatorname{Im} \bar{x}_{a},
$$

где

$$
\begin{gathered}
\bar{x}_{a}=\frac{F \Delta_{s}}{\Delta_{a} \Delta_{s}-D^{2} \delta^{2}}, \\
\Delta_{a}=\omega_{a 0}^{2}+D^{2} \alpha-\omega^{2}+i \eta_{a} \omega, \\
\Delta_{s}=\omega_{s 0}^{2}+D^{2} \beta-\omega^{2}+i \eta_{s} \omega .
\end{gathered}
$$

Здесь $\omega_{a 0}$ и $\omega_{s 0}-$ собственные частоты антисимметричных и симметричных колебаний в состоянии без нарушения симметрии $(D=0)$. Выражение $(8)$ в дальнейшем используется для расчета ИК спектров в состояниях с нарушенной симметрией $(D \neq 0)$.

\section{Дискуссия}

На рис. 2 и 3 представлены спектры поглощения характеристических колебаний и их изменение с ростом асимметрии молекулы для серии молекулярных параметров. Все параметры даны в единицах частоты антисимметричного колебания $\omega_{a 0}$ в состоянии без нарушения симметрии, значение которой обычно находится в интервале от 2000 до $2200 \mathrm{~cm}^{-1}$. Значения молекулярных параметров выбраны из условия, что наблюдаемые в экспериментах сдвиги частот полос поглощения, порождаемые нарушением симметрии, лежат в пределах нескольких десятков обратных сантиметров, что в относительных единицах составляет порядка одного процента. Это означает, что параметр малости в разложении по степеням параметра асимметрии (3) имеет порядок 0.01, что гарантирует высокую точность используемого приближения. Этот малый параметр позволяет также пренебречь влиянием квадратичного электронноколебательное взаимодействие $U_{\mathrm{int}}^{(2)}$ на нарушение симметрии. При выводе выражения для спектра поглощения (8) это проявилось в том, что параметр $D$ не зависит от параметров, входящих в $U_{\text {int }}^{(2)}$. В расчетах использовано значение однородной ширины рассматриваемых 

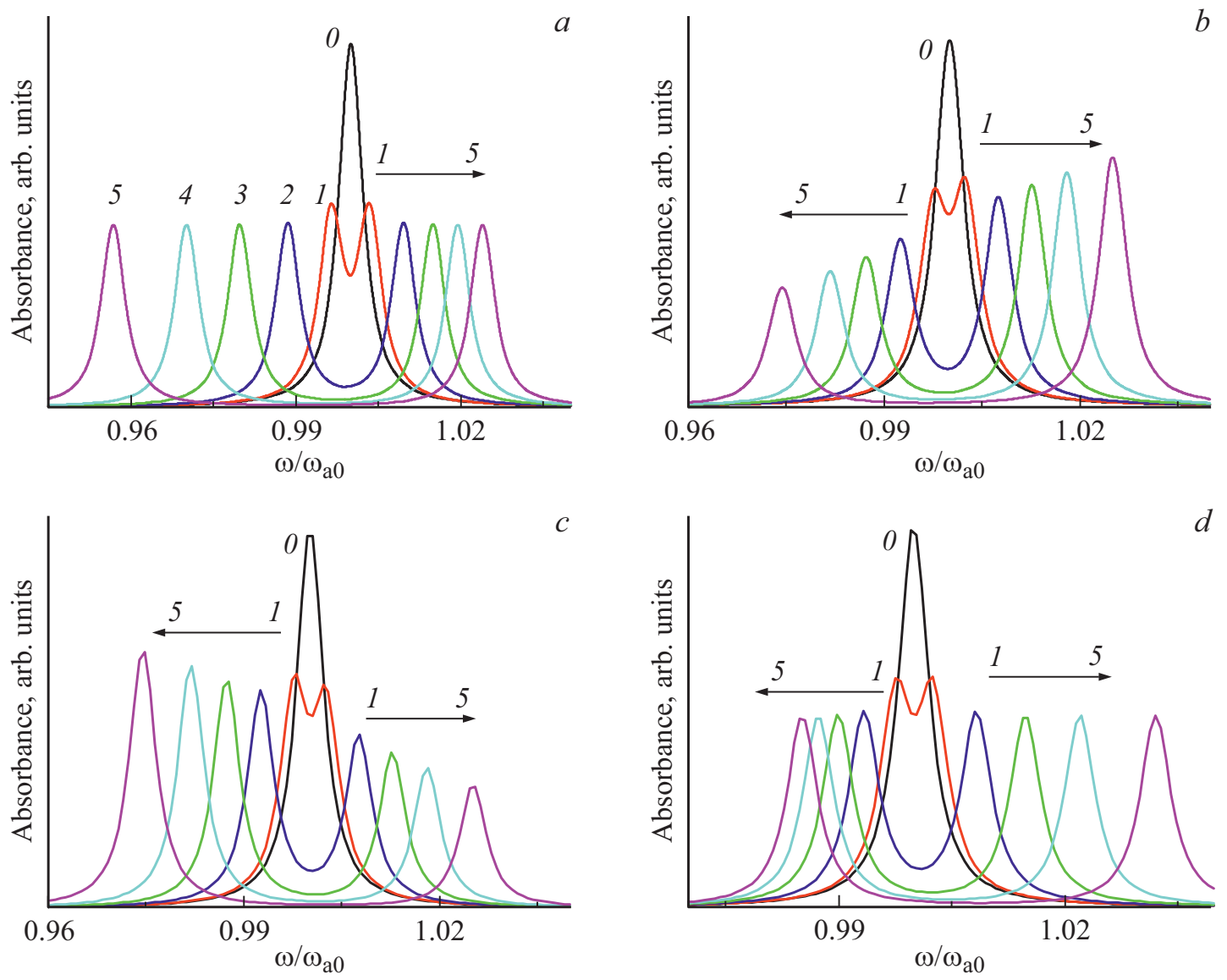

Рис. 2. Изменение ИК спектров поглощения характеристических колебаний с ростом степени асимметрии: $D=0(0)$, $0.1(1), 0.3(2), 0.5(3), 0.7(4), 0.95(5 \mid /)$. Значения не варьируемых параметров: $\omega_{a 0}=\omega_{s 0}, \eta_{a}=\eta_{s}=0.005 \omega_{a 0}, \delta / \omega_{a 0}=0.05$. Изменяющиеся параметры: $\alpha / \omega_{a 0}^{2}=-0.02, \beta / \omega_{a 0}^{2}=-0.02(a) ; \alpha / \omega_{a 0}^{2}=0.02, \beta / \omega_{a 0}^{2}=-0.02(b) ; \alpha / \omega_{a 0}^{2}=-0.02, \beta / \omega_{a 0}^{2}=0.02(c)$; $\alpha / \omega_{a 0}^{2}=0.02, \beta / \omega_{a 0}^{2}=0.02(d)$.

ИК полос, измеренных методами нестационарной 2D ИК спектроскопии [21]. Они приблизительно равны $\eta_{s} \cong \eta_{a}=10 \mathrm{~cm}^{-1}$.

Форма спектров и их изменения с ростом степени асимметрии молекулы критически зависят от соотношения частот $\omega_{a 0}$ и $\omega_{s 0}$ в состоянии без нарушения симметрии. Если частоты $\omega_{a 0}$ и $\omega_{s 0}$ очень близки друг к другу, то при малых значениях параметра $D$ полоса антисимметричного колебания расщепляется на две близкие по интенсивности полосы (рис. 2). Если частоты $\omega_{a 0}$ и $\omega_{s} 0$ различаются на хотя бы один процент, то нарушение симметрии сопровождается возникновением второй полосы, интенсивность которой растет с ростом асимметрии, начиная с нулевого значения (рис. 3). Именно такое поведение демонстрируют результаты экспериментов с молекулами вида A- $\pi$-D- $\pi$-A и D- $\pi$-A- $\pi$-D $[13,22]$. В обоих исследованиях оказалось, что частота антисимметричной моды меньше частоты симметричной $\omega_{a 0}<\omega_{s 0}$.

В исследованных экспериментально молекулах группы с тройными связями удалены друг от друга на большие расстояния, так что взаимодействие между ни- ми должно быть слабым. Вследствие этого естественно предположить, что частоты $\omega_{a 0}$ и $\omega_{s 0}$ совпадают и равны собственным частотам этих идентичных групп. Следует отметить, что частота асимметричных колебаний в возбужденном состоянии $\omega_{a 0}$ измеряется в экспериментах с высокой точностью, тогда как $\omega_{s 0}$ до сих пор не измерена, хотя принципиально это возможно, и такие эксперименты планируются. В основном состоянии молекул эти частоты измерялись, и оказалось, что в пределах экспериментальной ошибки частоты $\omega_{a 0}$ и $\omega_{s} 0$ совпадают. Из этих данных можно сделать важное заключение, что основным механизмом расщепления частот $\omega_{a 0}$ и $\omega_{s 0}$ является линейное электронно-колебательное взаимодействие (4), а ангармонические взаимодействия несущественны. Во-первых, ангармонические взаимодействия мод имеются в основном и возбужденном состояниях, и нет оснований полагать, что они существенно различаются. Расщепление наблюдается только в возбужденном состоянии. Во-вторых, ангармонические взаимодействия мод могут как увеличивать, так и уменьшать частоты $\omega_{a 0}$ и $\omega_{s 0}$, но на разную величину, что и приведет к их расщеплению. При этом возможны оба варианта: 

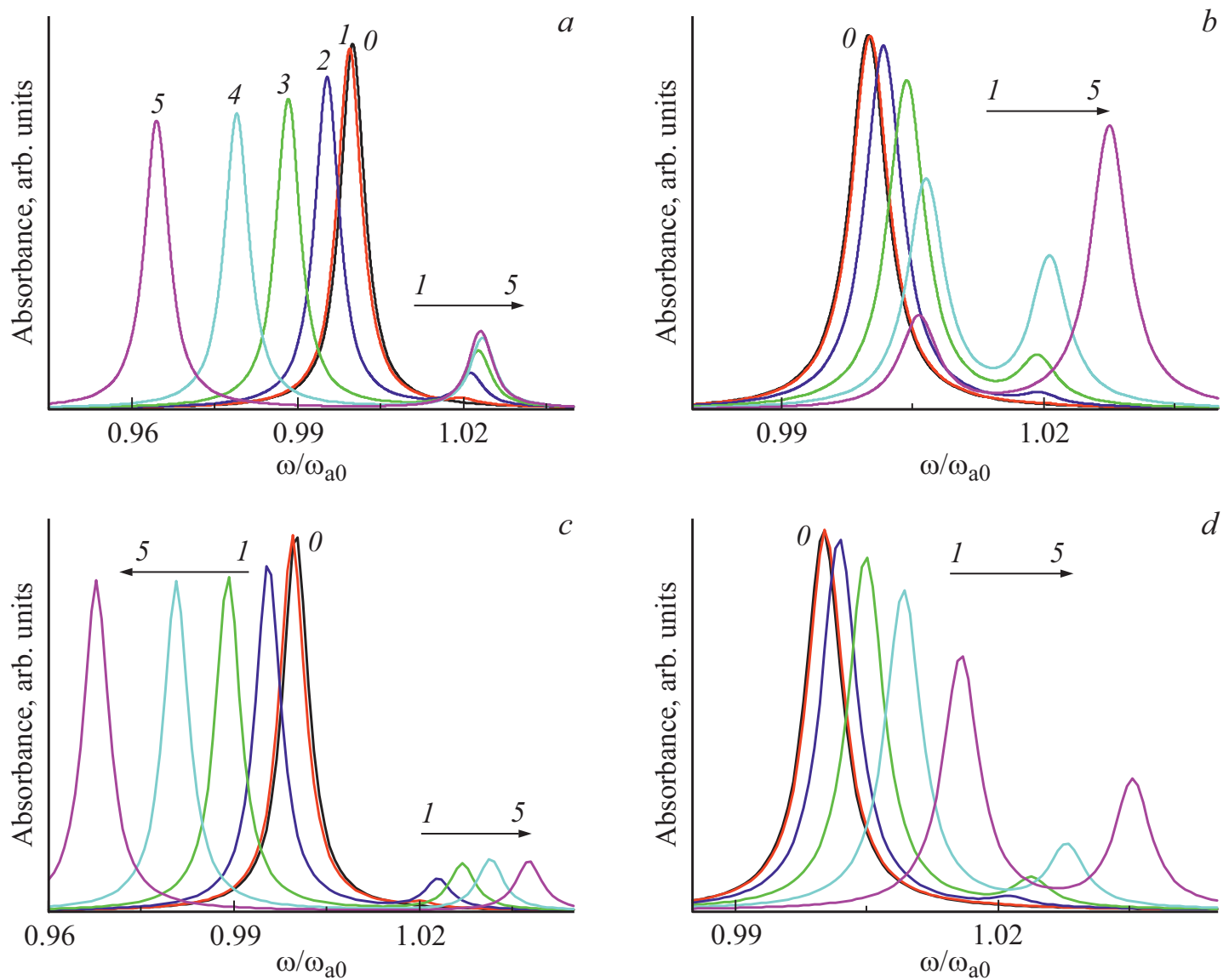

Рис. 3. Изменение ИК спектров поглощения характеристических колебаний с ростом степени асимметрии: $D=0(0), 0.1(1)$, 0.3 (2), 0.5 (3), 0.7 (4), 0.95 (5). Значения не варьируемых параметров: $\omega_{s 0}=1.02 \omega_{a 0}, \eta_{a}=\eta_{s}=0.005 \omega_{a 0}$. Изменяющиеся параметры: $\delta / \omega_{a 0}=0.05(a, c), 0.02(b, d) . \alpha / \omega_{a 0}^{2}=-0.05, \beta / \omega_{a 0}^{2}=-0.02(a) ; \alpha / \omega_{a 0}^{2}=0.05, \beta / \omega_{a 0}^{2}=-0.02(b) ; \alpha / \omega_{a 0}^{2}=-0.05$, $\beta / \omega_{a 0}^{2}=0.02(c) ; \alpha / \omega_{a 0}^{2}=0.05, \beta / \omega_{a 0}^{2}=0.02(d)$.

$\omega_{a 0}<\omega_{s 0}$ и $\omega_{a 0}>\omega_{s 0}$. Наблюдается только первый, хотя следует отметить, что имеющаяся в настоящее время выборка очень короткая. В то же время линейное электронно-колебательное взаимодействие предсказывает заметное понижение только частоты $\omega_{a 0}$ и только в возбужденном состоянии, в полном соответствии с экспериментальными данными.

Перейдем к более детальному анализу закономерностей изменения спектров с ростом параметра асимметрии $D$, представленных на рис. 2 и 3. В обсуждениях состояний с нарушенной симметрией мы пользуемся терминами симметричной и антисимметричной моды, имея в виду, что в данном подходе это базисные моды. В спектрах проявляются частоты нормальных колебаний, являющихся суперпозицией базисных.

Изменения спектров с ростом степени асимметрии молекулы контролируются тремя параметрами: $\alpha, \beta, \delta$. Частоты антисимметричного и симметричного колебаний в асимметричном состоянии испытывают сдвиги, которые определяются выражениями $\omega_{a}^{2}=\omega_{a 0}^{2}+D^{2} \alpha$ и $\omega_{s}^{2}=\omega_{s 0}^{2}+D^{2}$ соответственно. Между базисными модами есть взаимодействие, интенсивность которого зада- ется параметром $D \delta$. Это взаимодействие приводит к новым нормальным модам, расщепление собственных частот которых больше расщепления частот антисимметричной и симметричной мод.

На рис. 2 показаны типичные изменения ИК спектров поглощения с ростом параметра асимметрии $D$ при близких частотах $\omega_{a 0}=\omega_{s 0}$ для четырех возможных сочетаний знаков параметров $\alpha$ и $\beta$. В случае, когда параметры $\alpha$ и $\beta$ имеют одинаковый знак, низко- и высокочастотные полосы имеют близкие интенсивности при любых значениях параметра асимметрии (рис. 2, $a$ и $d$ ). Они совпадают при $\alpha=\beta$, и их различие невелико даже, когда $\alpha$ отличается от $\beta$ в два раза. Причина этого состоит в том, что расщепление частот базисных колебаний $\omega_{a}$ и $\omega_{s}$ слабо меняется с ростом $D$, и базисные моды дают примерно одинаковый вклад в каждое нормальное колебание. Значение расщепления частот нормальных колебаний, определяющее расстояние между полосами поглощения, контролируется единственным параметром $D \delta$. В случае, когда параметры $\alpha$ и $\beta$ имеют разные знаки, интенсивности низко- и высокочастотных полос сильно различаются (рис. $2, b$ и $c$ ). Причина 
состоит в том, что теперь расщепление частот базисных колебаний $\omega_{a}$ и $\omega_{s}$ нарастает с увеличением $D$, в результате вклады базисных мод в нормальные становятся разными. При большом расщеплении базисные моды смешиваются слабо, и нормальные моды приближаются по своему составу к базисным.

На рис. 3 представлены ИК спектры поглощения для случая, когда различие между $\omega_{a 0}$ и $\omega_{s 0}$ составляет $2 \%$. Здесь абсолютная величина параметра $\alpha$ превышает величину $\beta$ в два с половиной раза. Это означает, что антисимметричная мода сильнее симметричной откликается на нарушение симметрии. Это ожидаемо и соответствует имеющимся экспериментальным данным. Расщепление частот $\omega_{a 0}$ и $\omega_{s 0}$ ведет к сильному подавлению высокочастотной полосы. Чтобы на рис. 3, $a$ и $c$ эта полоса была ясно видна, параметр взаимодействия антисимметричной и симметричной мод увеличен до $\delta=0.05$. Физические причины, лежащие в основе этой закономерности, следующие. Расщепление частот $\omega_{a}$ и $\omega_{s}$ таково, что взаимодействие между ними $D \delta$ оказывается слабым, в результате одна нормальная мода включает антисимметричную моду с большим весом и поэтому сильно поглощает. Для второй нормальной моды картина обратная.

Спектры и их изменение с ростом асимметрии молекулы в случае с одинаковыми знаками параметров $\alpha$ и $\beta$ представлены на рис. $3, a$ и $d$. Поведение спектров на рис. 3, $a$ близко к наблюдаемым в экспериментах с молекулой A- $\pi$-D- $\pi$-A, включающей две $-\mathrm{C} \equiv \mathrm{N}-г$ руппы, в серии растворителей разной полярности $[11,13]$. Одна из особенностей этих спектров состоит в том, что частота максимума слабой полосы почти не меняется с ростом параметра асимметрии, а сильная полоса испытывает большой сдвиг. Это связано с тем, что два механизма, связанные с параметрами $\alpha$ и $\beta$, сдвигают сильную полосу в одном и том же направлении, тогда как сдвиги слабой полосы, обусловленные $\beta$ и $\delta$, почти компенсируют друг друга.

Изменения спектров, представленных на рис. 3, $a$ и $d$, существенно различаются тем, что с ростом $D$ интенсивность высокочастотной полосы на рис. 3, $a$ практически не изменяется и остается малой, а на рис. $3, d$ достигает больших значений. Причина этих отличий состоит в том, что в первом случае расщепление частот базисных колебаний растет, а во втором уменьшается. В результате сближения частот базисных колебаний с ростом $D$ интенсивность высокочастотной полосы достигает достаточно больших значений (рис. $3, d$ ).

Закономерности изменения спектров в случае с противоположенными знаками параметров $\alpha$ и $\beta$ представлены на рис. $3, b$ и $c$. Если частоты базисных колебаний $\omega_{a}$ и $\omega_{s}$ удаляются друг от друга с ростом параметра асимметрии (рис. 3,c), то интенсивность высокочастотной полосы остается малой. Причина этого состоит в том, что хотя с ростом $D$ взаимодействие базисных мод растет, параллельный рост разности частот $\omega_{a}$ и $\omega_{s}$ сохраняет малое смешивания этих мод. То есть, в нормальных модах всегда доминирует одна из базисных мод.

Качественно иная картина наблюдается в ситуации, когда частоты базисных колебаний $\omega_{a}$ и $\omega_{s}$ сближаются с ростом асимметрии (рис. $3, b)$. При достижении параметром асимметрии значения $D_{0}$, определяемого равенством

$$
D_{0}=\sqrt{\frac{\omega_{s 0}^{2}-\omega_{a 0}^{2}}{\alpha-\beta}},
$$

частоты антисимметричной и симметричной мод совпадают: $\omega_{a}=\omega_{s}$. Из уравнения (8) следует, что в этой точке интенсивности полос выравниваются. Естественно, чтобы эта точка была достижима в экспериментах, должно выполняться неравенство $D_{0}<1$. Дальнейший рост параметра $D$ приводит к тому, что при $D>D_{0}$ частота антисимметричной моды становится больше частоты симметричной моды: $\omega_{a}>\omega_{s}$, иинтенсивность высокочастотной полосы превышает интенсивность низкочастотной полосы. Таким образом, в области малых значений параметра асимметрии: $D<D_{0}$, с ростом $D$ полосы сближаются, а при $D>D_{0}$ они начинают разбегаться. Минимальное расстояние между полосами $\Delta \omega=D_{0} \delta / \omega_{a 0}$ достигается при $D=D_{0}$. Легко показать, что в этой и только в этой точке нормальными модами являются локальные колебания левой и правой ветвей молекулы. Для молекулы D-л-A- $\pi$-D, исследованной в работе [22], эта точка достижима в сильно полярных растворителях, что дает возможность прямого измерения частот локальных колебаний (валентных колебаний $-\mathrm{C} \equiv \mathrm{C}$-групп).

Поведение ИК спектра, подобное представленному на рис. $3, b$, наблюдалось в экспериментах с молекулой $\mathrm{D}-\pi-\mathrm{A}-\pi-\mathrm{D}$, включающей две $-\mathrm{C} \equiv \mathrm{C}$-группы [22]. В исследуемой системе нарушение симметрии протекало достаточно медленно и удалось зарегистрировать эволюцию спектров характеристических колебаний. С течением времени нарастало значение параметра асимметрии, что и приводило к наблюдаемым изменениям спектра [19]. Таким образом, экспериментальные данные свидетельствуют о том, что в молекуле с - $\mathrm{C} \equiv \mathrm{N}$ группами параметры $\alpha$ и $\beta$ одного знака, тогда как в молекуле $\mathrm{c}-\mathrm{C} \equiv \mathrm{C}$-группами они разного знака. Является ли это закономерностью, пока не известно, нужны дополнительные экспериментальные исследования на других молекулах.

В исследованных молекулах ИК полосы оказались достаточно узкими, так что выполняется неравенство $\eta_{s} \cong \eta_{a} \ll \omega_{a 0}$. В этом пределе из уравнения (8) получаем общее выражение для разности частот максимумов полос

$$
\Delta \omega=\frac{\sqrt{\left(\omega_{a}^{2}-\omega_{s}^{2}\right)^{2}+4 D^{2} \delta^{2}}}{2 \omega_{a} 0} .
$$

Зависимость $\Delta \omega$ от $D$ будет немонотонной, если $D_{0}<1$, и монотонной в противном случае. Обсуждаемая ранее молекула A- $\pi$-D-л-A имеет такие параметры, что расщепление полос характеризуется монотонной 
зависимостью и хорошо аппроксимируется простым выражением $\Delta \omega=a+b D^{2}$ с положительными постоянными $a$ и $b$, не зависящими от растворителя $[13,16]$. Поскольку параметр асимметрии $D$, а вместе с ним и расщепление $\Delta \omega$ очень чувствительны к значению энергии взаимодействия молекулы с растворителем [13], то это означает, что $\Delta \omega$ является мерой этой энергии взаимодействия. Если теперь измеренные значения $\Delta \omega$ в серии растворителей расположить в порядке их возрастания, то соответствующие им растворители тоже будут располагаться в порядке возрастания энергии их взаимодействия с молекулой. На качественном уровне этой шкалой можно пользоваться уже сейчас. В рамках модельных представлений возможно создание и количественной шкалы.

\section{Заключение}

В настоящейработе в рамках разработанной ранее теории нарушения симметрии переносом заряда в возбужденных квадрупольных молекулах и его проявлений в нестационарных ИК спектрах дана классификация типов возможных изменений ИК спектров с ростом степени асимметрии молекул. Дана физическая интерпретация предсказываемых закономерностей. Из 8 возможных типов изменений, представленных на рис. 2 и 3 , в экспериментах уже наблюдались 2 типа $[13,22]$. Отметим, что до настоящего времени исследованы только две молекулы.

В рассматриваемом подходе нормальными колебаниями являются четная и нечетная комбинации характеристических локальных колебаний. Учитывая, что группы, валентные колебания которых мы изучаем, разнесены на большое расстояние и поэтому очень слабо взаимодействуют друг с другом, можно предположить, что они взаимодействуют с излучением независимо, и в ИК спектрах видны полосы, соответствующие этим колебаниям. В этом случае должны были бы наблюдаться изменения спектров, похожие на представленные на рис. 2. При слабом нарушении симметрии эти группы очевидно имеют близкие дипольные моменты и интенсивности их полос поглощения тоже должны были быть близкими. Экспериментальные данные противоречат этому ожиданию и показывают, что вторая полоса при слабом нарушении симметрии имеет малую интенсивность в согласии с принятым здесь описанием.

\section{Финансирование работы}

Работа выполнена при финансовой поддержке Российского фонда фундаментальных исследований в рамках научного проекта № 19-03-00175.

\section{Конфликт интересов}

Авторы заявляют, что у них нет конфликта интересов.

\section{Список литературы}

[1] Albota M., Beljonne D., Bredas J.L., Ehrlich J.E., Fu J.Y., Heikal A.A., Hess S.E., Kogej T., Levin M.D., Marder S.R. et al. // Science. 1998. V. 281. P. 1653-1656. doi $10.1126 /$ science.281.5383.1653

[2] Le Droumaguet C., Mongin O., Werts M.H.V., BlanchardDesce M. // Chem. Commun. 2005. P. 2802-2804. https://doi.org/10.1039/B502585K

[3] Nowak-Krol A., Grzybowski M., Romiszewski J., Drobizhev M., Wicks G., Chotkowski M., Rebane A., Gorecka E., Gryko D.T. // Chem. Commun. 2013. V. 49. P. 8368-8370. http://dx.doi.org/10.1039/C3CC44728F

[4] Yao H., Okada T., Mataga N. // J. Phys. Chem. 1989. V. 93. P. 7388-7394. https://doi.org/10.1021/j100358a028

[5] Piet J.J., Schuddeboom W., Wegewijs B.R., Grozema F.C., Warman J.M. // J. Am. Chem. Soc. 2001. V. 123. P. 5337 5347. https://doi.org/10.1021/ja004341o

[6] Woo H.Y., Liu B., Kohler B., Korystov D., Mikhailovsky A., Bazan G.C. // J. Am. Chem. Soc. 2005. V. 127. P. 1472114729. https://doi.org/10.1021/ja052906g

[7] Strehmel B., Sarker A.M., Detert H. // ChemPhysChem. 2003. V. 4. P. 249-259. https://doi.org/10.1002/cphc. 200390041

[8] Katan C., Terenziani F., Mongin O., Werts M.H.V., Porrés L., Pons T., Mertz J., Tretiak S., BlanchardDesce M. // J. Phys. Chem. A. 2005. V. 109. P. 302-3037. https://doi.org/10.1021/jp044193e

[9] Pawlicki M., Collins H.A., Denning R.G., Anderson H.L. // Angew. Chem. Int. Ed. 2009. V. 48. P. 3244-3266. https://doi.org/10.1002/anie.200805257

[10] Dereka B., Rosspeintner A., Li Z. et al. // J. Am. Chem. Soc. 2016. V. 138. P. 4643-4649. https://doi.org/10.1021/jacs.6b01362

[11] Dereka B., Rosspeintner A., Krzeszewski M. et al. // Angew. Chem. Int. Ed. 2016. V. 55. P. 15624-15628. https://doi.org/10.1002/anie.201608567

[12] Dereka B., Rosspeintner A., Stezycki R. et al. // J. Phys. Chem. Lett. 2017. V. 8. P. 6029-6034. https://doi.org/10.1021/acs.jpclett.7b02944

[13] Dereka B., Vauthey E. // J. Phys. Chem. Lett. 2017. V. 8. P. 3927-3932. https://doi.org/10.1021/acs.jpclett.7b01821

[14] Terenziani F., Painelli A., Katan C. et al. // J. Am. Chem. Soc. 2006. V. 128. P. 15742-15755. https://doi.org/10.1021/ja064521j

[15] Ivanov A.I., Dereka B., Vauthey E. // J. Chem. Phys. 2017. V. 146. Art. no 164306. https://doi.org/10.1063/1.4982067

[16] Ivanov A.I., Tkachev V.G. // J. Chem. Phys. 2019. V. 151. Art. no 124309. https://doi.org/10.1063/1.5116015

[17] Ivanov A.I. // J. Phys. Chem. C. 2018. V. 122. P. 29165-29172. https://doi.org/10.1021/acs.jpcc.8b10985

[18] Назаров А.Е., Иванов А.И. // Журн. физ. химии. 2020. Т. 86. C. 1206-1214. doi 10.31857/S004445372008021X

[19] Nazarov A.E., Ivanov A.I., Vauthey E. // J. Phys. Chem. C. 2020. V. 124. P. 2357-2369. https://dx.doi.org/10.1021/acs.jpcc.9b10565

[20] Иванов А.И., Ткачёв В.Г. // Изв. РАН. Серия физическая. 2020. T. 84. № 5. C. 631-635. doi $10.31857 / \mathrm{S} 0367676520050130$

[21] Dereka B., Helbing J., Vauthey E. // Angew. Chem. Int. Ed. 2018. V. 57. P. $17014-17018$. https://doi.org/10.1002/anie.201808324.

[22] Söderberg M., Dereka B., Marrocchi A., Carlotti B., Vauthey E. // J. Phys. Chem. Lett. 2019. V. 10. P. 2944-2948. https://doi.org/10.1021/acs.jpclett.9b01024 